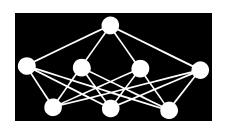

\title{
KINEMATICS ANALYSIS AND NUMERICAL SIMULATION OF HYBRID SERIAL-PARALLEL MANIPULATOR BASED ON NEURAL NETWORK
}

\author{
A. Rahmani, A. Ghanbari, M. Mahboubkhah
}

\begin{abstract}
This paper presents solution of kinematics analysis of a specific class of serial-parallel manipulators, known as 2-(6UPS) manipulators, which are composed of several modules consisting of elementary manipulators with the parallel structure of the Stewart platform, by artificial neural network. At first, the kinematics model of the hybrid manipulator is obtained. Then, as the inverse kinematics problem of this kind of manipulators is a very difficult problem to solve because of their highly nonlinear relations between joint variables and position and orientation of the end effectors, a wavelet based neural network (wave-net) with its inherent learning ability as a strong method was used to solve the inverse kinematics problem. Also, the proposed wavelet neural network (WNN) is applied to approximate the paths of middle and upper plate in a circular and a spiral path, respectively. The results show high accurate performance of the proposed WNN.
\end{abstract}

Key words: hybrid manipulators, kinematics analysis, network training, nonlinear system, wavelet neural network

Received: April 26, 2014

DOI: $10.14311 /$ NNW.2015.25.022

Revised and accepted: December 25, 2014

\section{Introduction}

A hybrid manipulation system is a sequence of parallel mechanisms which can overcome the limited workspace of parallel mechanism and can provide feature of both for serial and parallel mechanism. They are able to achieve high stiffness and high force-to-weight ratio. The hybrid serial-parallel robotic manipulator has attracted the attention of many researchers recently [19, 10, 7, 26], and it also has growing applications to robotics, machine tools, positioning systems, measurement devices, and so on. It has shown great potential and advantage both closed-loop and opened-loop manipulator over the traditional manipulator. Many different types of hybrid robots have been investigated [22, 4, 3, 28]. Tanio [24] presented

\footnotetext{
Arash Rahmani - Corresponding author, Ahmad Ghanbari, Mehran Mahboubkhah, Department of Mechanical Engineering, University of Tabriz, Tabriz, ZIP Code: 5166616471, Iran, Tel: +98 4136661496, E-mail: ara_rahmani@yahoo.com, ag3313656@gmail.com, ahboobkhah@tabrizu.ac.ir
} 
a hybrid (parallel-serial) manipulator consisting of two serially connected parallel mechanisms and overall 6-DOF and gave its closed-form solution for forward and inverse position problems. Romdhane [23] investigated the hybrid manipulator which was made of a base and two platforms in series and the motion of the mid platform is restricted only to three translations and the second platform rotates spherically with respect to the mid platform using joint connected the mid platform and top platform. The characteristics of 6-DOF parallel-serial hybrid manipulators which feature a 3-DOF in series actuated module mounted on the moving plate of another 3-DOF in parallel actuated manipulator with prismatic actuators is studied in [13]. The kinematics of hybrid type manipulation system with 6-DOF, which consists of a 3-DOF planar parallel platform and a 3-DOF serial robot arm, is discussed by Yang et al. [25]. Huang et al. [14] studied a conceptual design and dimensional synthesis of a 3-DOF parallel mechanism module which forms the main body of a newly invented 5-DOF reconfigurable hybrid robot. LiangZhi et al. [17] studied a hybrid 5-DOF manipulator based on the novel 3-RPS inactuated parallel manipulator. In their design a 2-DOF serial working table is placed over the mobile platform. A new methodology to synthesize hybrid robots as a whole structure is presented by Campos et al. [2]. Their method is based on Assure groups as the simplest basic blocks to build kinematic chains. Gallardo et al. [8] studied kinematics and dynamics of 2-(3-RPS) manipulators by means of screw theory and the principle of virtual work. A novel 3-RPS-3-SPR serial-parallel manipulator (S-PM) with 6 degree of freedoms is proposed in [11] and its inverse kinematics, active forces and workspace are solved. First, the inverse displacement is solved in close form based on the geometrical and the dimensional constraints. Then, Jacobian matrices are derived and the active forces are solved using the principle of virtual work. Gallardo et al. [9] address the kinematics, including position, velocity and acceleration analyses, of a modular spatial hyper-redundant manipulator built with a variable number of serially connected identical mechanical modules with autonomous motions. Li et al. [16] used a hybrid manipulator as a multi-dimensional vibration isolator based on the parallel mechanism. The scheme design, inverse kinematics, workspace and dexterity are carried out in their paper. Kizir et al. [15] used Kane transition function to generate several trajectories for controlling a high precision hybrid platform by a PID and sliding mode controller. Chen et al. [5] proposed a multi-objective genetic algorithm trajectory planner for a PKM, based on the dynamics approach. Parikh et al. [18] presented iteration neural network strategy for solution of forward kinematic of parallel mechanisms.

In this paper, a novel hybrid robot 2-(6-UPS) is introduced that comprises a sequence of two identical Stewart mechanism modules. The serial form of these hybrid manipulators overcomes the limited workspace of parallel manipulators and improves overall stiffness and response characteristics. Then, kinematic model of the hybrid robot is presented and because highly nonlinear relations between joint variables and position and orientation of the end effectors, wavelet neural network has been provided and used to solve the forward kinematics. In addition, the proposed WNN is applied to approximate the paths of mid and upper plate in circle and spiral path respectively. 


\section{Description of the hybrid robot}

The mechanism under investigation in this paper consists of two identical modules (base and upper modules) such that each module is Stewart Platform mechanism with 6-DOFs. In this hybrid mechanism, we have three platforms and twelve pods. Base platform is stationary and connected to middle platform via 6 extensible pods. Also, middle platform is connected to upper platform (as an end effecter) via 6 extensible pods. Each pod connects to the platform at its connection point through a spherical joint, and to the base at its connection point through universal joint. Each pod consists of two parts: the upper part and the lower part, which connect to each other through prismatic joint. Therefore, it is referred to as the 2-(6UPS) mechanism. This manipulator is actuated by motors located on the prismatic joints. Fig. 1 shows the design of the mentioned hybrid robot and structure of the middle and upper plates. Cartesian coordinate frame is attached to each plate with $\mathrm{z}$ axis normal to the plate.

\section{Forward and inverse kinematics solution}

Mechanism kinematics deals with the study of the mechanism motion as constrained by the geometry of the links. Typically, the study of mechanism kinematics is divided into two parts: inverse kinematics and direct kinematics. In the about mentioned hybrid robot, the inverse kinematics problem involves mapping a known pose (position and orientation) of the moving platforms of the mechanism to a length of each module's pods. The direct kinematics problem involves the mapping from a known length of each module's pods to a pose of the moving platforms. In this section, the inverse and forward kinematics problems of proposed mechanism are described in closed form.
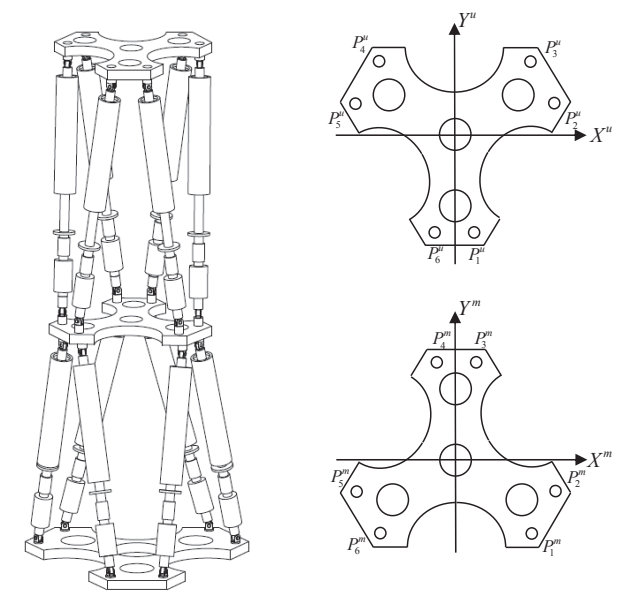

Fig. 1 Configuration of 2-(6-UPS) hybrid manipulator and plates.

The proposed kinematics model (vectorial kinematic analysis) using in this paper has some advantages compared to other methods: 
a) This method provides a geometric description of rigid motion which greatly simplifies the analysis of mechanism [21]. So, it is easily generalized to kinematic analysis for most hybrid parallel-parallel, parallel-serial and serial-serial manipulators.

b) This method suffers from singularity problems less than differential kinematics based solution and it is useful and more applicable for work space analysis [20].

c) This method can be used easily for mechanism velocity mapping especially for parallel and hybrid manipulators.

Fig. 2 shows the vectorial representation of the $i$-th pod at each module. According to Fig. 2, the middle and upper moving platforms frame are shown by $\left\{\mathbf{O}^{\mathrm{m}}\right\}$ and $\left\{\mathbf{O}^{\mathrm{u}}\right\}$, respectively, and base frame with $\left\{\mathbf{O}^{\mathrm{b}}\right\}$. Also, $\left(x^{\mathrm{m}}, y^{\mathrm{m}}, z^{\mathrm{m}}, \theta_{x}^{\mathrm{m}}, \theta_{y}^{\mathrm{m}}, \theta_{z}^{\mathrm{m}}\right)$ and $\left(x^{\mathrm{u}}, y^{\mathrm{u}}, z^{\mathrm{u}}, \theta_{x}^{\mathrm{u}}, \theta_{y}^{\mathrm{u}}, \theta_{z}^{\mathrm{u}}\right)$ present the location (position and orientation) of the middle and upper moving platform respectively. Now, the inverse kinematics of each module is obtained at first, and then forward kinematics is considered. The inverse kinematic problem of the platforms involves determination of the linear position, of six Pods for each module through considering a specified position, of the middle and upper moving platforms center.

For each module, homogeneous transformation matrices from frame $\left\{\mathbf{O}^{\mathrm{u}}\right\}$ to frame $\left\{\mathbf{O}^{\mathrm{m}}\right\}$ and frame $\left\{\mathbf{O}^{\mathrm{m}}\right\}$ to frame $\left\{\mathbf{O}^{\mathrm{b}}\right\}$ are $\mathbf{H}_{O^{\mathrm{u}}, O^{\mathrm{m}}}$ and $\mathbf{H}_{O^{\mathrm{m}}, O^{\mathrm{b}}}$ respectively, and are described as:

$$
\begin{aligned}
& \mathbf{H}_{O^{\mathrm{u}}, O^{\mathrm{m}}}=\left[\begin{array}{llll}
R_{1}^{\mathrm{u}} & R_{4}^{\mathrm{u}} & R_{7}^{\mathrm{u}} & x^{\mathrm{u}} \\
R_{2}^{\mathrm{u}} & R_{5}^{\mathrm{u}} & R_{8}^{\mathrm{u}} & y^{y} \\
R_{3}^{\mathrm{u}} & R_{6}^{\mathrm{u}} & R_{9}^{\mathrm{u}} & z^{\mathrm{u}} \\
0 & 0 & 0 & 1
\end{array}\right]= \\
& =\left[\begin{array}{llll}
C \theta_{z}^{\mathrm{u}} C \theta_{y}^{\mathrm{u}} & -S \theta_{z}^{\mathrm{u}} C \theta_{x}^{\mathrm{u}}+C \theta_{z}^{\mathrm{u}} S \theta_{y}^{\mathrm{u}} S \theta_{x}^{\mathrm{u}} & S \theta_{z}^{\mathrm{u}} S \theta_{x}^{\mathrm{u}}+C \theta_{z}^{\mathrm{u}} S \theta_{y}^{\mathrm{u}} C \theta_{x}^{\mathrm{u}} & x^{\mathrm{u}} \\
S \theta_{z}^{\mathrm{u}} C \theta_{y}^{\mathrm{u}} & C \theta_{z}^{\mathrm{u}} C \theta_{x}^{\mathrm{u}}+S \theta_{z}^{\mathrm{u}} S \theta_{y}^{\mathrm{u}} S \theta_{x}^{\mathrm{u}} & -C \theta_{z}^{\mathrm{u}} S \theta_{x}^{\mathrm{u}}+S \theta_{z}^{\mathrm{u}} S \theta_{y}^{\mathrm{u}} C \theta_{x}^{\mathrm{u}} & y^{y} \\
-S \theta_{y}^{\mathrm{u}} & C \theta_{y}^{\mathrm{u}} S \theta_{x}^{\mathrm{u}} & C \theta_{y}^{\mathrm{u}} C \theta_{x}^{\mathrm{u}} & z^{\mathrm{u}} \\
0 & 0 & 0 & 1
\end{array}\right], \\
& \mathbf{H}_{O^{\mathrm{m}}, O^{\mathrm{b}}}=\left[\begin{array}{llll}
R_{1}^{\mathrm{m}} & R_{4}^{\mathrm{m}} & R_{7}^{\mathrm{u}} & x^{\mathrm{m}} \\
R_{2}^{\mathrm{m}} & R_{5}^{\mathrm{m}} & R_{8}^{\mathrm{u}} & y^{\mathrm{m}} \\
R_{3}^{\mathrm{m}} & R_{6}^{\mathrm{m}} & R_{9}^{\mathrm{u}} & z^{\mathrm{m}} \\
0 & 0 & 0 & 1
\end{array}\right]= \\
& =\left[\begin{array}{llll}
C \theta_{z}^{\mathrm{m}} C \theta_{y}^{\mathrm{m}} & -S \theta_{z}^{\mathrm{m}} C \theta_{x}^{\mathrm{m}}+C \theta_{z}^{\mathrm{m}} S \theta_{y}^{\mathrm{m}} S \theta_{x}^{\mathrm{m}} & S \theta_{z}^{\mathrm{m}} S \theta_{x}^{\mathrm{m}}+C \theta_{z}^{\mathrm{m}} S \theta_{y}^{\mathrm{m}} C \theta_{x}^{\mathrm{m}} & x^{\mathrm{m}} \\
S \theta_{z}^{\mathrm{m}} C \theta_{y}^{\mathrm{m}} & C \theta_{z}^{\mathrm{m}} C \theta_{x}^{\mathrm{m}}+S \theta_{z}^{\mathrm{m}} S \theta_{y}^{\mathrm{m}} S \theta_{x}^{\mathrm{m}} & -C \theta_{z}^{\mathrm{m}} S \theta_{x}^{\mathrm{m}}+S \theta_{z}^{\mathrm{m}} S \theta_{y}^{\mathrm{m}} C \theta_{x}^{\mathrm{m}} & y^{\mathrm{m}} \\
-S \theta_{y}^{\mathrm{m}} & C \theta_{y}^{\mathrm{m}} S \theta_{x}^{\mathrm{m}} & C \theta_{y}^{\mathrm{m}} C \theta_{x}^{\mathrm{m}} & z^{\mathrm{m}} \\
0 & 0 & 0 & 1
\end{array}\right],
\end{aligned}
$$

where $C \theta=\cos (\theta), S \theta=\sin (\theta)$.

Beside, ${ }^{O^{\mathrm{m}}} \mathbf{L}_{i}^{\mathrm{u}}$ and ${ }^{O^{\mathrm{b}}} \mathbf{L}_{i}^{\mathrm{u}}$ are the vectors of length of the $i$-th pod in upper module relative to $\mathbf{O}^{\mathrm{m}}$ and $\mathbf{O}^{\mathrm{b}}$ frame respectively. And, $O^{\mathrm{b}} \mathbf{L}_{i}^{\mathrm{b}}$ is the vector of length of 
Rahmani A., Ghanbari A., Mahboubkhah M.: Kinematics analysis and...

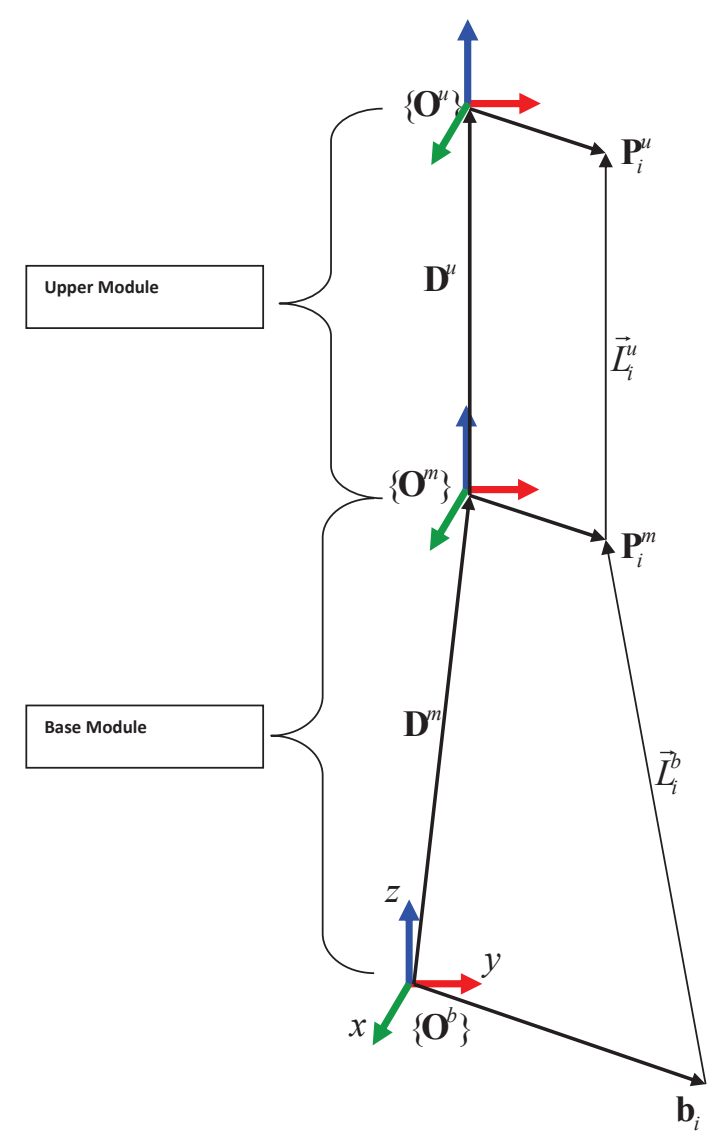

Fig. 2 Vectorial representation of the $i$-th pod at each module.

the $i$-th pod in base module relative to $\mathbf{O}^{\mathrm{b}}$ frame. Vector of length of the $i$-th pod without considering modules and frames, is a $1 \times 4$ vector which is described as

$$
\mathbf{L}_{i}=\left(\mathrm{L}_{i x}, \mathrm{~L}_{i y}, \mathrm{~L}_{i z}, 1\right)
$$

Also, $\mathbf{b}_{i}, \mathbf{P}_{i}^{\mathrm{m}}, \mathbf{P}_{i}^{\mathrm{u}}$ are the six vertices of the base, middle and upper plates relative to $\mathbf{O}^{\mathrm{b}}, \mathbf{O}^{\mathrm{m}}$ and $\mathbf{O}^{\mathrm{u}}$ frame respectively:

$$
\begin{array}{ll}
\mathbf{b}_{i}=\left(b_{i x}, b_{i y}, 0,1\right) & i=1,2, \ldots, 6, \\
\mathbf{P}_{i}^{\mathrm{m}}=\left(p_{i x}^{\mathrm{m}}, p_{i y}^{\mathrm{m}}, 0,1\right) & i=1,2, \ldots, 6, \\
\mathbf{P}_{i}^{\mathrm{u}}=\left(p_{i x}^{\mathrm{u}}, p_{i y}^{\mathrm{u}}, 0,1\right) & i=1,2, \ldots, 6 .
\end{array}
$$

Now, according to transformation of a vector from one frame to another, we can determine ${ }^{O^{\mathrm{b}}} \mathbf{L}_{i}^{\mathrm{b}}, O^{\mathrm{m}} \mathbf{L}_{i}^{\mathrm{u}}$ and ${ }^{O^{\mathrm{b}}} \mathbf{L}_{i}^{\mathrm{u}}$ as

$$
O^{\mathrm{b}} \mathbf{L}_{i}^{\mathrm{b}}=\left(\mathbf{H}_{O^{\mathrm{m}}, O^{\mathrm{b}}} \mathbf{P}_{i}^{\mathrm{m}}-\mathbf{b}_{i}\right),
$$




$$
\begin{gathered}
O^{\mathrm{m}} \mathbf{L}_{i}^{\mathrm{u}}=\left(\mathbf{H}_{O^{\mathrm{u}}, O^{\mathrm{m}}} \mathbf{P}_{i}^{\mathrm{u}}-\mathbf{P}_{i}^{\mathrm{m}}\right), \\
O^{\mathrm{b}} \mathbf{L}_{i}^{\mathrm{u}}=\mathbf{H}_{O^{\mathrm{m}}, O^{\mathrm{b}}}{ }^{\mathrm{m}} \mathbf{L}_{i}^{\mathrm{u}}=\mathbf{H}_{O^{\mathrm{m}}, O^{\mathrm{b}}}\left(\mathbf{H}_{O^{\mathrm{u}}, O^{\mathrm{m}}} \mathbf{P}_{i}^{\mathrm{u}}-\mathbf{P}_{i}^{m}\right) .
\end{gathered}
$$

Scalar form of each pod's length for each module is as

$$
\begin{array}{ll}
l_{i}^{\mathrm{u}}=\left\|O^{\mathrm{b}} \mathbf{L}_{i}^{\mathrm{u}}\right\|=\sqrt{O^{\mathrm{b}} L_{i x}^{\mathrm{u}}+O^{\mathrm{b}} L_{i y}^{\mathrm{u}}+O^{\mathrm{b}} L_{i z}^{\mathrm{u}}} & i=1, \ldots, 6, \\
l_{i}^{\mathrm{b}}=\left\|O^{\mathrm{b}} \mathbf{L}_{i}^{\mathrm{b}}\right\|=\sqrt{O^{\mathrm{b}} L_{i x}^{\mathrm{b}}+O^{\mathrm{b}} L_{i y}^{\mathrm{b}}+O^{\mathrm{b}} L_{i z}^{\mathrm{b}}} & i=1, \ldots, 6 .
\end{array}
$$

Eqs. (7) and (9) define the length of pods of each module relative to base in a closed form as a vector. Mapping the pose of middle and upper plates to the length of pods is easy and straightforward. But, because of highly nonlinear characteristic of these equations, the mapping from a known length of each module's pods to a pose of the moving platforms is rather difficult and too complicated. Therefore, wavelet based neural network (wave-net) is applied to solve forward kinematics of this mechanism. As it will be seen in the next, we use length of each pod of each module (Eqs. (10) and (11)) as input data for training the proposed neural network.

\section{Wavelet neural network}

\subsection{Structure of network}

The wavelet neural network (WNN) is the model based on wavelet transformation and artificial neural network [27]. Due to wavelet transform, it has good localization characteristics in time and frequency domain and neural networks have good ability to approximate complicated maps, WNN incorporate the good learning ability and the good property of localization, which have been successfully applied in function approximation and pattern classification. A neural network is constructed by interconnecting a number of neurons so as to form a network in which all connections are made in the forward direction.

Since the approximation class is nonlinear in the adjustable parameters, the training procedure of the neural networks may become trapped in some local minimum depending on the initialization. To overcome this problem, the wavelet networks have been proposed as an alternative to neural networks, which follow the availability of rates of convergence for approximation by wavelet based networks.

In this section, a feed-forward single hidden layer network is introduced. For a (back propagation) BP neural network with only one hidden layer of neurons, using basis wavelets as it's activate functions of hidden layer, we get a multi-input and multi-output wavelet neural networks in Fig. 3.

This WNN has $m, p, n$ nodes in the input layer, hidden layer and output layer, respectively. And the activate function of the $j$-th node in the hidden layer is [1]

$$
\psi_{a_{j}, b_{j}}(t)=\frac{1}{\sqrt{a_{j}}} \psi\left(\frac{t-b_{j}}{a_{j}}\right) \quad j=1,2, \ldots, p,
$$

where $\psi(t)$ is the mother wavelet function which is localized both in time and frequency and could be chosen as a different function according to the feature of 
the problem. Fig. 4 shows four types of most applicable wavelet functions. In this paper we use the Mexican Hat wavelet. This wavelet is derived from a function, which is proportional to the second derivative of the Gaussian probability density function. It is non-orthogonal, with infinite support and has maximum energy around origin with a narrow band. The expression for the Mexican Hat wavelet is given by Eq. (13). In this paper, $f(t)$ is chosen as sigmoid function:

$$
\psi(t)=\left(1-2 t^{2}\right) \cdot \exp \left(-t^{2}\right)
$$

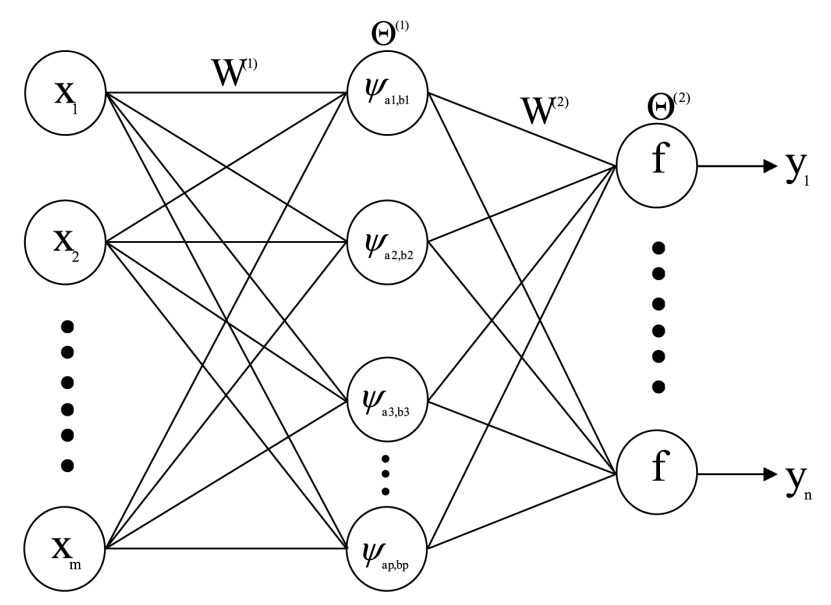

Fig. 3 Structure of $W N N$.

The wavelet neural network parameters in Fig. $3,\left(W^{(1)}, W^{(2)}, \Theta^{(1)}, \Theta^{(2)}, a_{1}, \ldots\right.$, $\left.a_{p}, b_{1}, \ldots, b_{p}\right)$, should be adjusted through training.

\subsection{Training of network}

Back propagation method is the most frequently used technique for training a feed forward network. It involves two passes through the network, a forward pass and a backward pass. The forward pass generates the network's output activities and the backward pass involves propagating the error initially found in the output nodes back through the network to assign errors to each node that contributed to the initial error. Once all the errors are assigned, the weights are changed so as to minimize these errors. Since the WNN in Fig. 3 is derived from a feed forward neural network, we use back propagation method to train this network. For the WNN in Fig. 3, when the input vector is $\mathbf{X}=\left(x_{k}\right)=\left(x_{1}, x_{2}, \ldots, x_{m}\right)$, we get the output of the $j$-th node in hidden layer:

$$
\psi_{a_{j}, b_{j}}\left(\sum_{k=1}^{\mathrm{m}} w_{j k}^{(1)} x_{k}-\theta_{j}^{(1)}\right)=\psi_{a_{j}, b_{j}}\left(F_{j}^{(1)}\right)=\frac{1}{\sqrt{a_{j}}} \psi\left(\frac{F_{j}^{(1)}-b_{j}}{a_{j}}\right)
$$




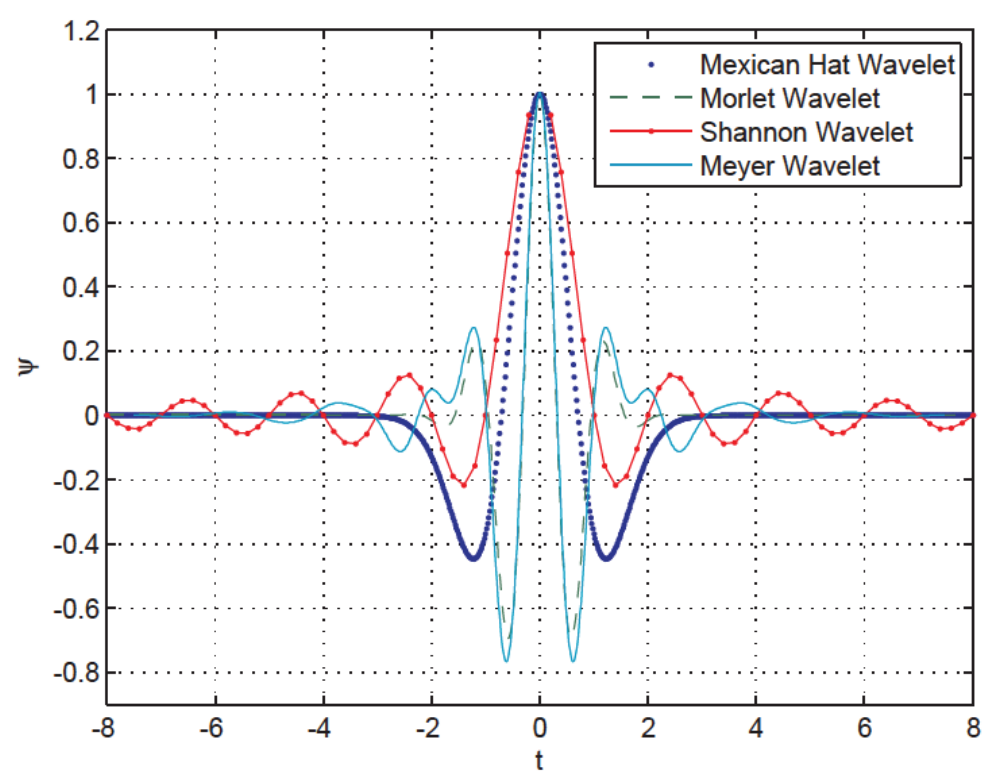

Fig. 4 Wavelet functions.

where

$$
F_{j}^{(1)}=\sum_{k=1}^{m}\left(w_{j k}^{(1)} x_{k}-\theta_{j}^{(1)}\right) .
$$

In this research, $m=6$ for each module and $\mathbf{X}=\left(x_{k}\right)=\left(x_{1}, x_{2}, \ldots, x_{6}\right)=$ $\left(l_{1}, l_{2}, \ldots, l_{6}\right)$.

The output of the $i$-th node of output layer is:

$$
y_{i}=f\left(\sum_{j=1}^{p} w_{i j}^{(2)} \psi_{a j, b j}\left(F_{j}^{(1)}\right)-\theta_{i}^{(2)}\right)=f\left(F_{i}^{(2)}\right),
$$

where

$$
F_{i}^{(2)}=\sum_{j=1}^{p}\left(w_{i j}^{(2)} \psi_{a j, b j}\left(F_{j}^{(1)}\right)-\theta_{i}^{(2)}\right) .
$$

From Eq. (14) we get the output vector of the WNN: Y $=\left(y_{1}, y_{2}, \ldots, y_{n}\right)$, which here is $(x, y, z, \alpha, \beta, \gamma)$ for each module. Suppose we have $Q$ training samples. For each sample $q$, the desired output vector is $\overline{\mathbf{Y}}_{q}=\left(\bar{y}_{q 1}, \bar{y}_{q 2}, \ldots, \bar{y}_{q n}\right)$, the output vector of the WNN is $\mathbf{Y}_{q}=\left(y_{q 1}, y_{q 2}, \ldots, y_{q n}\right)$. With these $\mathrm{Q}$ training samples, we train the WNN through batch learning process. Then the main goal of the network is to minimize the total error $E$ of each output node $i$ over all training samples $[12]:$

$$
E=\frac{1}{2} \sum_{q=1}^{Q} \sum_{i=1}^{n}\left(\bar{y}_{q i}-y_{q i}\right)^{2}
$$


By the iterative gradient descent method, the parameters of the wavelet neural network can be formulated by

$$
\begin{gathered}
w_{i j}^{(2)}(t+1)=(1+\beta) w_{i j}^{(2)}(t)-\beta w_{i j}^{(2)}(t-1)-\lambda \frac{\partial E}{\partial w_{i j}^{(2)}}, \\
w_{j k}^{(1)}(t+1)=(1+\beta) w_{j k}^{(1)}(t)-\beta w_{j k}^{(1)}(t-1)-\lambda \frac{\partial E}{\partial w_{j k}^{(1)}}, \\
\theta_{i}^{(2)}(t+1)=(1+\beta) \theta_{i}^{(2)}(t)-\beta \theta_{i}^{(2)}(t-1)-\lambda \frac{\partial E}{\partial \theta_{i}^{(2)}}, \\
\theta_{j}^{(1)}(t+1)=(1+\beta) \theta_{j}^{(1)}(t)-\beta \theta_{j}^{(1)}(t-1)-\lambda \frac{\partial E}{\partial \theta_{j}^{(1)}} \\
a_{j}(t+1)=(1+\beta) a_{j}(t)-\beta a_{j}(t-1)-\lambda \frac{\partial E}{\partial a_{j}} \\
b_{j}(t+1)=(1+\beta) b_{j}(t)-\beta b_{j}(t-1)-\lambda \frac{\partial E}{\partial b_{j}},
\end{gathered}
$$

where $t$ is the iteration index of learning and $\lambda$ is the learning rate. To improve the rate of learning, we modify the original learning rule with the momentum factor $\beta(0<\beta<1)$ to the weights [6]. The partial derivatives of the error $E$ with respect to each parameter can be calculated easily.

\section{WNN solution for kinematics of robot}

In order to model forward kinematics of hybrid robot with wave-net according to structure of robot, we have modeled the base module and the upper one, respectively. The input data of the network are the length of pods for each module according to Eqs. (9) and (10). At first and before proposing neural network, using inverse kinematics (mapping position and orientation of center point of mid and upper plate to length of pods), we generate some data for training the networks. Then the length of pods of the base module, $\mathbf{L}^{\mathrm{b}}=\left(l_{1}^{\mathrm{b}}, l_{2}^{\mathrm{b}}, l_{3}^{\mathrm{b}}, l_{4}^{\mathrm{b}}, l_{5}^{\mathrm{b}}, l_{6}^{\mathrm{b}}\right)$, is fed to the network to define the position and orientation of the middle plate, $\mathbf{P}^{\mathrm{m}}=\left(x^{\mathrm{m}}, y^{\mathrm{m}}, z^{\mathrm{m}}, \alpha^{\mathrm{m}}, \beta^{\mathrm{m}}, \gamma^{\mathrm{m}}\right)$. Repeating same steps as described in below, leads us to solving forward kinematics of upper module, too. For this, using the length of pods of the upper module, $\mathbf{L}^{\mathrm{u}}=\left(l_{1}^{\mathrm{u}}, l_{2}^{\mathrm{u}}, l_{3}^{\mathrm{u}}, l_{4}^{\mathrm{u}}, l_{5}^{\mathrm{u}}, l_{6}^{\mathrm{u}}\right)$, and the position and orientation of the middle plate, $\mathbf{P}^{\mathrm{m}}=\left(x^{\mathrm{m}}, y^{\mathrm{m}}, z^{\mathrm{m}}, \alpha^{\mathrm{m}}, \beta^{\mathrm{m}}, \gamma^{\mathrm{m}}\right)$, we calculated the position and orientation of the upper plate (end effecter), $\mathbf{P}^{\mathrm{u}}=\left(x^{\mathrm{u}}, y^{\mathrm{u}}, z^{\mathrm{u}}, \alpha^{\mathrm{u}}, \beta^{\mathrm{u}}, \gamma^{\mathrm{u}}\right)$. Therefore, we have two different networks.

The algorithm of the wavelet neural network for approximating the kinematics of hybrid robot for each module is summarized as follows:

Step 1: Set the initial values of networks parameters $\left(W^{(1)}, W^{(2)}, \Theta^{(1)}, \Theta^{(2)}, a_{1}\right.$, $\left.\ldots, a_{p}, b_{1}, \ldots, b_{p}\right)$, learning rate $\lambda$ and momentum factor $\beta$. 
Step 2: Input the training data and the desired output values. Give input vectors $\mathbf{X}=\left(x_{1}, x_{2}, \ldots, x_{m}\right)$ where it is the length of pods of each module and a desired output vector $\overline{\mathbf{Y}}_{q}=\left(\bar{y}_{q 1}, \bar{y}_{q 2}, \ldots, \bar{y}_{q n}\right)$, the theoretical values acquired from inverse kinematic solution of Eqs. (7) and (9).

Step 3: For each input datum, calculate the output of the wavelet neural network by Eq. (16).

Step 4: Adjust the networks parameters $\left(W^{(1)}, W^{(2)}, \Theta^{(1)}, \Theta^{(2)}, a_{1}, \ldots, a_{p}, b_{1}, \ldots\right.$, $\left.b_{p}\right)$ using gradient descent algorithm by Eqs. (19) to $(24)$.

Step 5: The error function $E$ is calculated by Eq. (18). If the error is less than the desired bound, the networks parameters are obtained and the learning process is terminated, else go to step 2.

\section{Results}

In this section, we present the results of the proposed WNN on approximating the kinematic analysis of hybrid robot. The architecture used for the wavelet network was one input layer with six neurons, one hidden layer with 153 neurons and one output layer with six neurons. The network was trained with a learning rate of 0.15 , a momentum term of 0.05 , and 2,048 learning iterations. The largest error $E$ or given precision is $0.5 \%$. Tab. I shows sample results of direct kinematics with reference length of pods $\left(L_{0}=550 \mathrm{~mm}\right)$ and pods length variation of base module and upper module:

$$
\begin{aligned}
& \mathbf{L}_{\text {base }}=\left(l_{1}=240 \mathrm{~mm}, l_{2}=50 \mathrm{~mm}, l_{3}=110 \mathrm{~mm}, l_{4}=190 \mathrm{~mm}, l_{5}=230 \mathrm{~mm}\right. \\
&\left.l_{6}=300 \mathrm{~mm}\right) \\
& \mathbf{L}_{\text {upper }}=\quad\left(\begin{array}{l}
\left(l_{1}=210 \mathrm{~mm}, l_{2}=140 \mathrm{~mm}, l_{3}=290 \mathrm{~mm}, l_{4}=260 \mathrm{~mm}, l_{5}=120 \mathrm{~mm}\right. \\
\left.l_{6}=40 \mathrm{~mm}\right) .
\end{array}\right.
\end{aligned}
$$

And a comparison of WNN with closed form solutions (CFS) is given there.

Also, Figs. 5 to 8 show the results of the proposed WNN for the specific paths of middle and upper plates of hybrid robot. Results given here are for circle path and spiral path with elliptical base curve for center point of middle and upper plate, respectively. Using inverse kinematic analysis for proposed paths of plates, we define motions of each pod of each module (Fig. 5). Then, we feed the proposed WNN by the pods motions to get the paths of plates. Fig. 6 shows the position paths and Fig. 7 shows orientation paths for center point of each plate. Again, we used the outputs of the proposed WNN for inverse kinematic analysis to define the new motions of each pod of each module and compared them with the pods motions which results from CFS. Fig. 8 show the results, for variation of length of each pods of base and upper module for circle path for center point of mid plate with $\left(10 \times\left[-\cos t^{\circ}, \sin t^{\circ}, 0^{\circ}\right]\right)$ orientation and spiral path with elliptical base curve for center point of upper plate with $\left(10 \times\left[\cos t^{\circ},-\sin t^{\circ}, 0^{\circ}\right]\right)$ orientation. 
Rahmani A., Ghanbari A., Mahboubkhah M.: Kinematics analysis and...

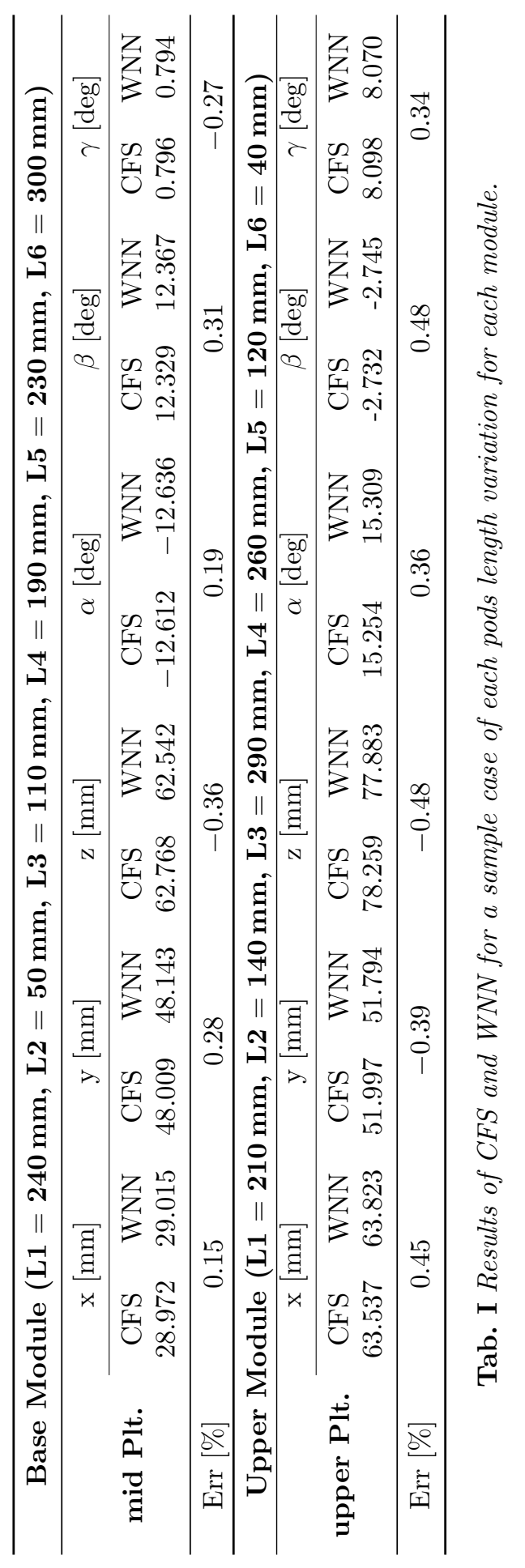




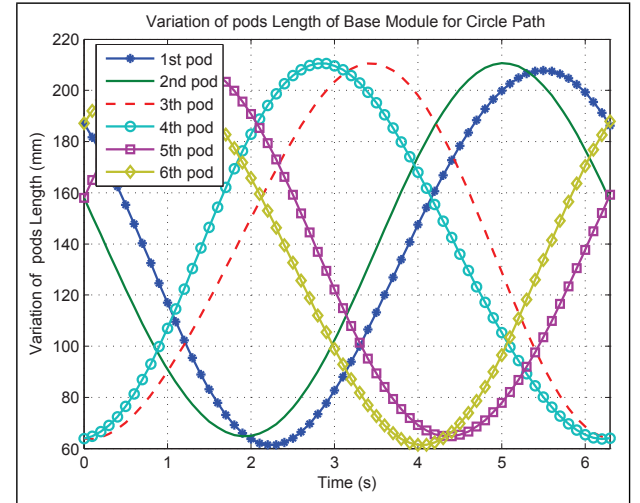

(a)

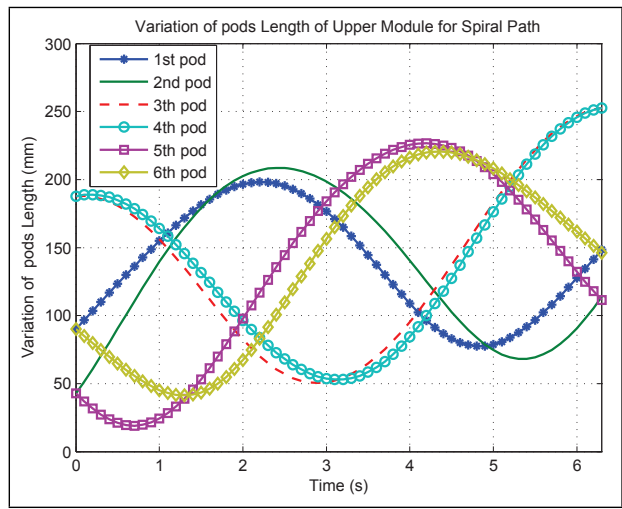

(b)

Fig. 5 (a) Variation of length of pods of base module for circle path with orientation $\left(10 \times\left[-\cos t^{\circ}, \sin t^{\circ}, 0^{\circ}\right]\right),(b)$ Variation of length of pods of upper module for spiral path with orientation $\left(10 \times\left[\cos t^{\circ},-\sin t^{\circ}, 0^{\circ}\right]\right)$.

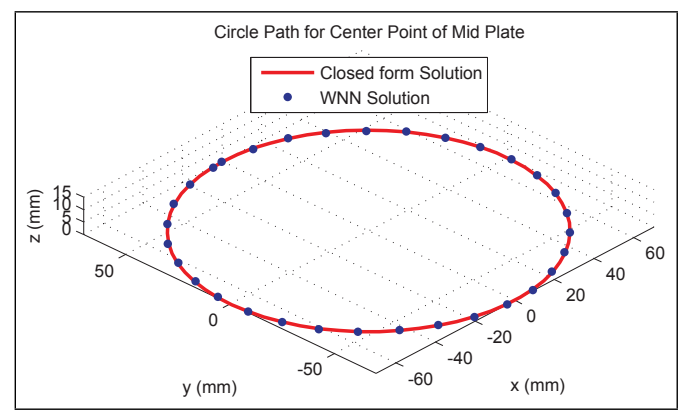

(a)

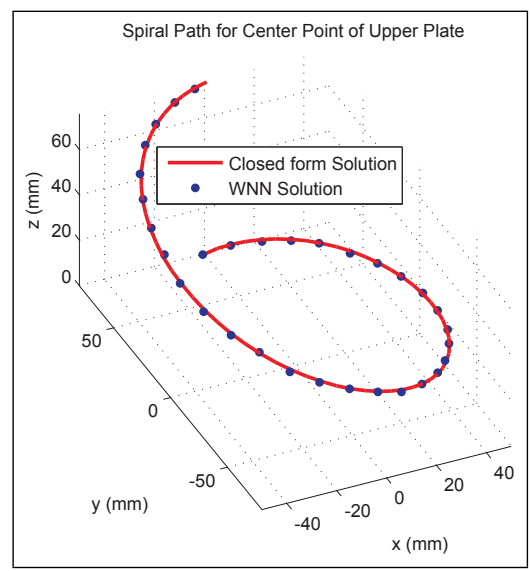

(b)

Fig. 6 (a) Circle path for center point of mid plate with orientation $(10 \times$ $\left.\left[-\cos t^{\circ}, \sin t^{\circ}, 0^{\circ}\right]\right)$, (b) Spiral path for center point of upper plate with orientation $\left(10 \times\left[\cos t^{\circ},-\sin t^{\circ}, 0^{\circ}\right]\right)$. 
Rahmani A., Ghanbari A., Mahboubkhah M.: Kinematics analysis and...

The results show good agreement between exact solution (CFS) and outputs of proposed WNN. However, the accumulation of error for kinematic analysis of upper module causes the error percentage in results of upper plate position and orientation is higher than mid plate. Accumulation of error for kinematic analysis of upper module is derived:

1. Network error,

2. Input error (the outputs of WNN for base module with error, are also fixed inputs for WNN for upper plates).

\section{Conclusions}

In this paper, WNN method was employed to solve forward kinematics of a hybrid robot. The proposed network can be proved to have the capability of approximating any multivariable systems. Whereas the kinematic model of hybrid robot has

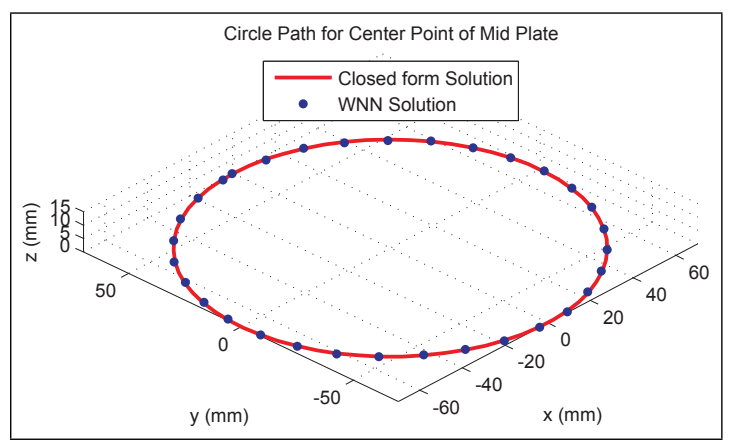

(a)

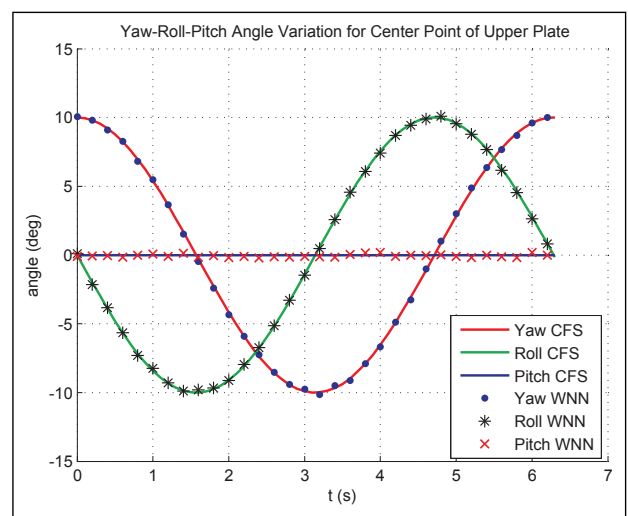

(b)

Fig. 7 (a) Orientation path for center point of mid plate, (b) Orientation path for center point of upper plate. 


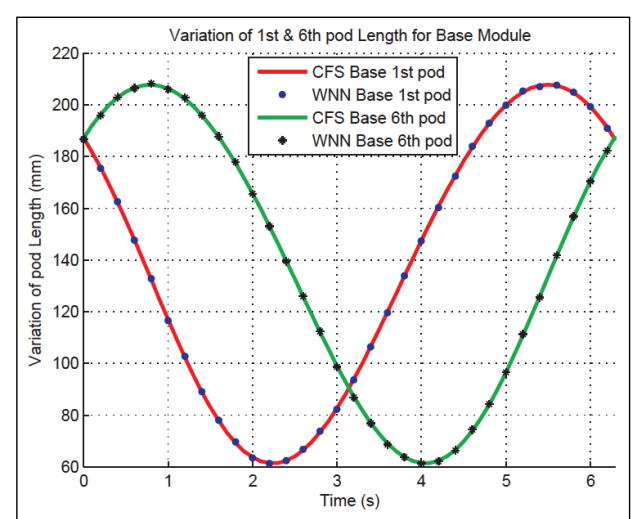

(a)

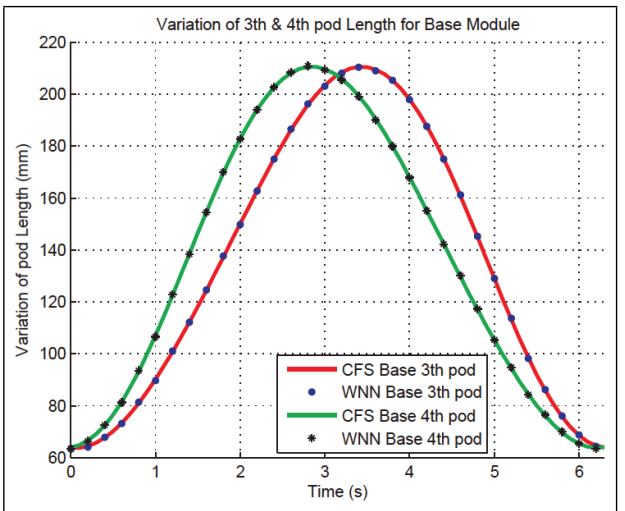

(c)

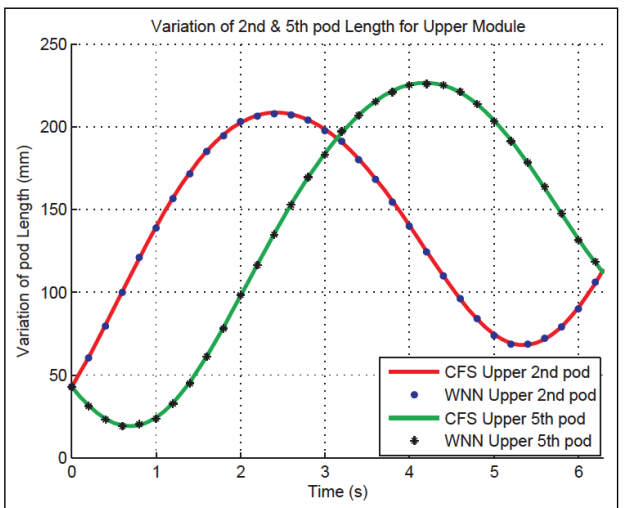

(e)

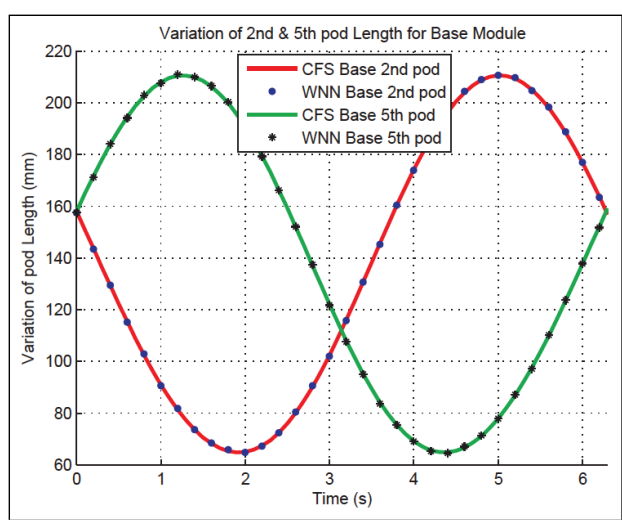

(b)

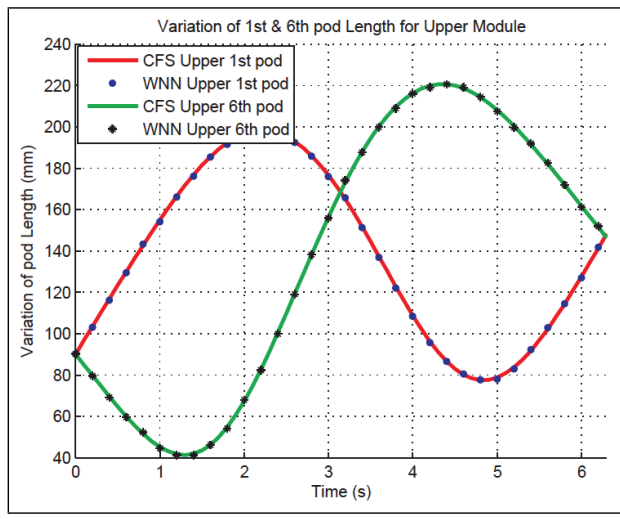

(d)

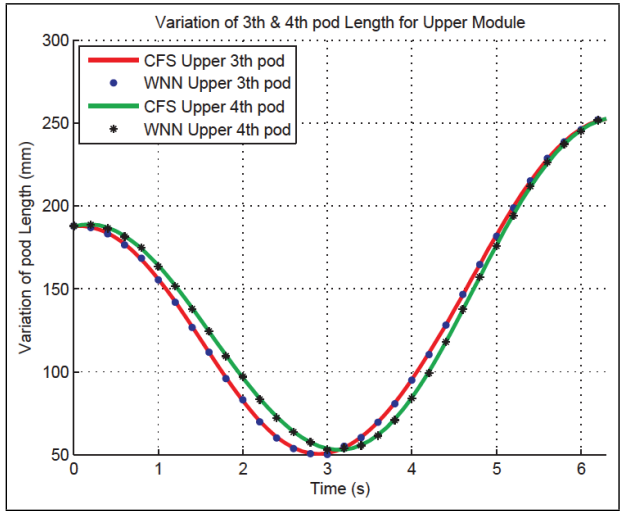

(f)

Fig. 8 (a)-(f) Length variation of pods of base and upper module for circle and spiral path respectively with defined orientation change. 
Rahmani A., Ghanbari A., Mahboubkhah M.: Kinematics analysis and...

strongly nonlinear characteristic, the network can not only be trained in a short time, but also shows better performance in solving problems. According to the results, there is good agreement between WNN and CFS, but, because of accumulation of error, the error of results of upper plate is larger than the error of the mid plate. Nevertheless, according networks tanning approach the maximum error for all cases is less than $0.5 \%$.

\section{References}

[1] BANAKAR A., AZEEM M.F. Artificial wavelet neuro-fuzzy model based on parallel wavelet network and neural network. Soft Computing. 2008, 12(8), pp. 789-808, doi: 10.1007/s00500-007-0238-z.

[2] CAMPOS A., BUDDE C., HESSELBACH J. A type synthesis method for hybrid robot structures. Mechanism and Machine Theory. 2008, 43(8), pp. 984-995, doi: 10.1016/j.mechmachtheory.2007.07.006.

[3] CARBOnE G., CECCARELLI M. A stiffness analysis for a hybrid parallel-serial manipulator. Robotica. 2004, 22(5), pp. 567-576, doi: 10.1017/S0263574704000323.

[4] CARBONE G., CECCARELLI M. A serial-parallel robotic architecture for surgical tasks. Robotica. 2005, 23(3), pp. 345-354, doi: 10.1017/S0263574704000967.

[5] CHEN C.-T., PHAM H.-V. Trajectory planning in parallel kinematic manipulators using a constrained multi-objective evolutionary algorithm. Nonlinear Dynamics. 2012, 67(2), pp. 1669-1681, doi: 10.1007/s11071-011-0095-2.

[6] CHEN D., HAN J. Application of wavelet neural network in signal processing of MEMS accelerometers. Microsystem technologies. 2011, 17(1), pp. 1-5, doi: 10.1007/s00542-010-1169-7.

[7] COPPOLA G., ZHANG D., LIU K. A 6-DOF reconfigurable hybrid parallel manipulator. Robotics and Computer-Integrated Manufacturing. 2014, 30(2), pp. 99-106, doi: 10.1016/j.rcim.2013.09.011.

[8] GALlARDO-ALVARADO J., AGUILAR-NÁJERAA C.R., CASIQUE-ROSASA L., RICOMARTÍNEZ J.M., ISLAM N. Kinematics and dynamics of 2 (3-RPS) manipulators by means of screw theory and the principle of virtual work. Mechanism and Machine Theory. 2008, 43(10), pp. 1281-1294, doi: 10.1016/j.mechmachtheory.2007.10.009.

[9] GALLARDO J., LESSO R., RICO J.M., ALICI G. The kinematics of modular spatial hyperredundant manipulators formed from RPS-type limbs. Robotics and Autonomous Systems. 2011, 59(1), pp. 12-21, doi: 10.1016/j.robot.2010.09.005.

[10] HU B. Formulation of unified Jacobian for serial-parallel manipulators. Robotics and Computer-Integrated Manufacturing. 2014, 30(5), pp. 460-467, doi: 10.1016/j.rcim.2014.03.001.

[11] HU B., HU B., LU Y., YU J.J., ZHUANG S. Analyses of Inverse Kinematics, Statics and Workspace of a Novel 3RPS-3SPR Serial-Parallel Manipulator. The Open Mechanical Engineering Journal. 2012, 5, pp. 65-72, doi: 10.2174/1874155X01206010065.

[12] HUANG M., CUI B. A novel learning algorithm for wavelet neural networks. Advances in Natural Computation. 2005, Springer, pp. 1-7, doi: 10.1007/11539087_1.

[13] HUANG M.Z., LING S.-H., SHENG Y. A study of velocity kinematics for hybrid manipulators with parallel-series configurations. In: Proceedings of the IEEE International Conference on Robotics and Automation, Atlanta, GA. IEEE, 1993, 1, pp. 456 - 461, doi: 10.1109/ROBOT.1993.292022.d.

[14] HUANG T., LI M., ZHAO X.M., MEI J.P., CHETWYND D.G., HU S.J. Conceptual design and dimensional synthesis for a 3-DOF module of the TriVariant-a novel 5-DOF reconfigurable hybrid robot. IEEE Transactions on Robotics. 2005, 21(3), pp. 449-456, doi: 10.1109/TRO.2004.840908. 
[15] KIZIR S., BINGUL Z. Position Control and Trajectory Tracking of the Stewart Platform. In: S. KUCUK, ed. Serial and Parallel Robot Manipulators - Kinematics, Dynamics, Control and Optimization. Rijeka: InTech, 2012, pp. 179-202, doi: 10.5772/35569.

[16] LI B., ZHAO W., DENG Z. Modeling and analysis of a multi-dimensional vibration isolator based on the parallel mechanism. Journal of Manufacturing Systems. 2012, 31(1), pp. 50-58, doi: $10.1016 /$ j.jmsy.2010.12.001.

[17] LIANGZHi F., ELATTA A., XIAOPING L. Kinematic calibration for a hybrid 5-DOF manipulator based on 3-RPS in-actuated parallel manipulator. The International Journal of Advanced Manufacturing Technology. 2005, 25(7-8), pp. 730-734, doi: 10.1007/s00170-003-1987-1.

[18] PARIKH P.J., LAM S.S. Solving the forward kinematics problem in parallel manipulators using an iterative artificial neural network strategy. The International Journal of Advanced Manufacturing Technology. 2009, 40(5-6), pp. 595-606, doi: 10.1007/s00170-007-1360-x.

[19] PISLA D., GHERMAN B., VAIDA C., SUCIU M., PLITEA, N. An active hybrid parallel robot for minimally invasive surgery. Robotics and Computer-Integrated Manufacturing. 2013, 29(4), pp. 203-221, doi:10.1016/j.rcim.2012.12.004.

[20] QAZANI M.R.C., PEDRAMMEHR S., RAHMANI A., DANAEI B., ETTEFAGH M.M., RAJAB A.K.S., ABDI H. Kinematic analysis and workspace determination of hexarot-a novel 6-DOF parallel manipulator with a rotation-symmetric arm system. Robotica. pp. 118, doi: 10.1017/S0263574714000988.

[21] QAZANI M.R.C., PEDRAMMEHR S., RAHMANI A., SHAHRYARI M., RAJAB A.K.S., ETTEFAGH M.M. An experimental study on motion error of hexarot parallel manipulator. The International Journal of Advanced Manufacturing Technology. 2014, 72(9-12), pp. 13611376, doi: 10.1007/s00170-014-5685-y.

[22] RICO J., GALLARDO J., DUFFY J. Screw theory and higher order kinematic analysis of open serial and closed chains. Mechanism and Machine Theory. 1999, 34(4), pp. 559-586, doi: 10.1016/S0094-114X(98)00029-9.

[23] ROMDHANE L. Design and analysis of a hybrid serial-parallel manipulator. Mechanism and Machine Theory. 1999, 34(7), pp. 1037-1055, doi: 10.1016/S0094-114X(98)00079-2.

[24] TANEV T.K. Kinematics of a hybrid (parallel-serial) robot manipulator. Mechanism and Machine Theory. 2000, 35(9), pp. 1183-1196, doi: 10.1016/S0094-114X(99)00073-7.

[25] YANG G., CHEN W., HO E.H.L. Design and kinematic analysis of a modular hybrid parallelserial manipulator. In: Proceedings of the 7th International Conference on Control, Automation, Robotics and Vision (ICARCV 2002), Marina Mandarin, Singapore. IEEE, 2002, 1, pp. 45-50, doi: 10.1109/ICARCV.2002.1234788.

[26] ZENG Q., EHMANN K.F. Design of parallel hybrid-loop manipulators with kinematotropic property and deployability. Mechanism and Machine Theory. 2014, 71, pp. 1-26, doi: 10.1016/j.mechmachtheory.2013.08.017.

[27] ZHANG Q., BENVENISTE A. Wavelet networks. IEEE Transactions on Neural Networks. 1992, 3(6), pp. 889-898.

[28] ZHOU B., XU Y. Robust control of a 3-DOF hybrid robot manipulator. The International Journal of Advanced Manufacturing Technology. 2007, 33(5-6), pp. 604-613, doi: 10.1007/s00170-006-0474-x. 